\section{Análisis de los apoyos que reciben los jóvenes con discapacidad intelectual en su transición a la vida adulta en España: una investigación a partir de experiencias de profesionales y personas con discapacidad ${ }^{1}$}

Analysis of support received by young people with intellectual disabilities in their transition to adulthood in Spain: an investigation based on the experiences of professionals and people with disabilities

\footnotetext{
Resumen

El objetivo de este trabajo es obtener información acerca de la adecuación de la red de dispositivos o servicios donde estos jóvenes son objeto de acciones socioeducativas en su proceso de tránsito a la edad adulta en España. Se han aplicado entrevistas en profundidad a 45 profesionales y a 20 personas con discapacidad intelectual y el método Delphi a dos paneles integrados por 20 expertos cada uno. Los resultados muestran la escasa continuidad de los apoyos que reciben los jóvenes con discapacidad a lo largo de su proceso de tránsito y cuestionan la formación marcadamente académica que reciben los jóvenes como preparación su vida adulta. También se analiza la participación de los jóvenes y sus familias en el proceso. La investigación nos permite disponer de una visión contrastada y amplia del proceso de tránsito a la vida adulta de los jóvenes con discapacidad y plantear estrategias de mejora de este proceso.
}

\begin{abstract}
The objective of this study is to gather information regarding the adaptation of the range of socio educational projects and services aimed at the transition to an adult life of young persons with intellectual disabilities in Spain. The research of the study has been done in three stages. During each stage, a specific tool has been used. One to one in-depth interviews have been undertaken with 45 professionals and 20 individuals with intellectual disabilities. The Delphi method has been applied to two panels consisting of 20 experts each. Firstly, results focus on the approach to different issues related to the devices. Secondly, the training opportunities that these individuals receive to ease the transition period is addressed. And finally, the study refers to the participation of the individuals themselves and their families in the process. The developed analysis allows us to propose strategies to improve the transition to adult life.
\end{abstract}

\author{
Maria Pallisera \\ <maria.pallisera@udg.edu> \\ Profesora Titular. Departamento de \\ Pedagogía. Universidad de Girona

\section{Judit Fullana} \\ <judit.fullana@udg.edu> \\ Profesora Titular. Departamento de \\ Pedagogía. Universidad de Girona
}

\section{Montserrat Vilà}

<montserrat.vila@udg.edu>

Profesora Titular. Departamento de Pedagogía. Universidad de Girona

\section{Paco Jiménez}

<paco.jimenez@udg.edu>

Catedrático. Departamento de Pedagogía. Universidad de Girona

\section{Montse Castro}

<montse.castro@udg.edu>

Profesora asociada. Universidad de Girona

\section{Carolina Puyalto}

<carolina.puyalto@udg.edu>

Educadora social y psicopedagoga. Becaria de Investigación (FPI).

Universidad de Girona

\section{Mario Montero}

<mario.montero@udg.edu>

Profesor Asociado. Departamento de Pedagogía. Universidad de Girona

\section{Raquel Martin}

<raquelmartinpazos@hotmail.com>

Técnica de apoyo a la investigación. Grupo de Investigación en Diversidad. Universidad de Girona

\section{Para citar:}

Pallisera, M. et al. (20I4): "Análisis de los apoyos que reciben los jóvenes con discapacidad intelectual en su transición a la vida adulta en España: una investigación a partir de experiencias de profesionales y personas con discapacidad", Revista Española de Discapacidad, 2 (2): 27-43.

<http://dx.doi.org/IO.5569/2340$5104.02 .02 .02>$

Fecha de recepción: 22-05-20I4 Fecha de aceptación: 05-II-20I4 


\section{Palabras clave}

Transición a la edad adulta, personas con discapacidad intelectual, entrevistas, estudio Delphi, investigación cualitativa, sistema educativo, educación no formal.

\section{Keywords}

Transition to adulthood, people with intellectual disabilities, interviews, Delphi study, qualitative research, education system, non-formal education.

\section{La transición a la vida adulta de las personas con discapacidad intelectual: complejidad y necesidad de estudio}

El tema de la transición a la edad adulta y vida activa de las personas con discapacidad ha sido foco de estudio en los últimos 30 años. Constituye un proceso encaminado a lograr objetivos relacionados con la participación en actividades formativas más allá de la educación obligatoria, la inclusión laboral, el inicio de la emancipación de la familia, la implicación y participación activa en distintos espacios de la comunidad, y la posibilidad de experimentar relaciones sociales y personales satisfactorias (Cobb y Alwell, 2009; Kaehne y Beyer, 2009; Martínez Rueda, 2002; Test et al., 2009; Trainor, 2008). Las trayectorias individuales de transición que llevan a conseguir estos objetivos se construyen fundamentalmente en base a la articulación de apoyos que se ofrecen en servicios escolares y postescolares. Estudios realizados en distintos contextos (Hudson, 2006; Jordán de Urríes y Verdugo, 20I3; Kaehne y Beyer, 2009; Pallisera, 20 I Ia; Pallisera et al., 20I 2 y 20I4; Rosselló y Verger, 2008; Winn y Hay, 2009) ponen de manifiesto que actualmente la transición a la vida adulta sigue siendo uno de los procesos más complejos a los que se enfrentan los jóvenes con discapacidad intelectual.
La complejidad de la transición acentúa la necesidad de diseñar e implementar sistemas de apoyo a los jóvenes con discapacidad intelectual con el fin de ayudarles a desarrollar itinerarios que tomen en consideración los distintos roles que desempeñan las personas adultas en la sociedad. Es fundamental tener en cuenta que los procesos de tránsito se desarrollan en una red de dispositivos institucionales o servicios concretos, por lo que la comprensión en profundidad de la relación entre estos dispositivos se configura como un elemento primordial para poder emprender acciones que incidan en la optimización de la transición a la edad adulta y vida activa de las personas con discapacidad. Para ello, es necesario emprender estudios que tengan en cuenta de forma global las distintas dimensiones y escenarios implicados en este proceso (Hudson, 2006).

En los últimos años se han desarrollado en nuestro país trabajos que aportan datos relevantes sobre algunas dimensiones específicas de la transición. En este sentido, mencionamos una interesante investigación, centrada en la dimensión laboral, que se orienta a evaluar la calidad de las acciones de transición escuela-trabajo con el colectivo de personas con discapacidad y a proponer estrategias de mejora para el empleo (Fernández e Iglesias, 2005). Este trabajo, a partir del análisis de casos constituidos por programas, analiza la adecuación de dichos programas de formación y concluye con datos relativos a los elementos a establecer en el ámbito de los programas de formación ocupacional e intermediación laboral para facilitar el logro del empleo de las personas con discapacidad.

Otros estudios se han desarrollado con la finalidad de profundizar en las potencialidades del trabajo con apoyo como metodología de inclusión laboral (Becerra et al., 201 2; Egido et al., 2009; Jordán de Urríes et al., 2005; Jordán de Urríes y Verdugo, 2010; Vilà et al., 2007; Rius, 2005).

Centradas en la dimensión educativa, se configuran diversas líneas de estudio: por una parte, encontramos un conjunto de trabajos 
centrados en el papel de la escuela secundaria en el proceso de transición de los jóvenes con discapacidad intelectual (Pallisera et al., 2012; Vilà et al., 2010; Vilà et al., 2012). Estos trabajos apuntan algunas estrategias para asegurar un proceso inclusivo en el tránsito a la vida activa de los alumnos con discapacidad intelectual, tanto a nivel de políticas educativas como en relación con el despliegue de determinadas acciones psicopedagógicas. Por otra parte, en los últimos años están cobrando interés los estudios centrados en los llamados programas educativos de segunda oportunidad que, en sentido amplio, se refieren a una variada gama de prácticas educativas y formativas cuya finalidad es luchar contra la exclusión social ligada a la exclusión escolar (Adame y Salvà, 20IO). En este sentido, la investigación, aún incipiente, está mostrando las posibilidades de esta modalidad formativa para la capacitación de los jóvenes en situación de vulnerabilidad (Olmos y Mas, 2013) y, específicamente, de jóvenes con discapacidad (Castro, 20I3; Castro y Vilà, 20I3).

Ahora bien, para plantear estrategias y acciones concretas a diferentes niveles (normativo, institucional-organizativo, curricular y de articulación de servicios) facilitadoras de los procesos de transición se debe ir más allá de los estudios que se centren en una única dimensión (por ejemplo, la laboral) o escenario (por ejemplo, el sistema educativo). Por el contrario, es imprescindible abordar un enfoque o perspectiva global multidimensional que tenga en cuenta de forma completa el sistema o red de dispositivos en el que se construyen y desarrollan los procesos de tránsito. Además, esta perspectiva de análisis debe incluir como fuente de información a las propias personas con discapacidad: el conocimiento sobre cómo mejorar los recorridos personales hacia la vida adulta desde una óptica inclusiva se enriquecerá en la medida en que se incluya de forma efectiva a las personas con discapacidad en la toma de decisiones de los procesos de investigación. Es necesario buscar formas para garantizar que las personas con discapacidad intelectual estén correctamente representadas en las distintas fases de la investigación; para ello, deben promoverse proyectos colaborativos elaborados con rigor que equilibren puntos de vista y otorguen un mayor alcance de la investigación (Gill, I999; Walmsley y Johnson, 2003).

Con este planteamiento, se desarrolla la investigación que presentamos en este artículo, que tiene como finalidad conocer en profundidad cómo se produce en la actualidad el tránsito a la edad adulta y vida activa de los jóvenes con discapacidad intelectual en España, para plantear propuestas de actuación que mejoren sus oportunidades de conseguir objetivos relacionados con la inclusión laboral y social en la vida adulta. En concreto, se presentan los principales resultados del primer objetivo de esta investigación, el cual se propone la obtención de información acerca de la adecuación de la red de dispositivos o servicios donde los jóvenes con discapacidad intelectual son objeto de acciones socioeducativas en su proceso de transición a la edad adulta y vida activa en España.

\section{Método}

Para la consecución del objetivo citado, se plantea el estudio de la red de centros o servicios relacionados con el tránsito a la vida adulta de los jóvenes con discapacidad intelectual, con el objeto de detectar los principales problemas con los que se enfrentan estos jóvenes para realizar su transición desde una perspectiva inclusiva.

La investigación se realiza a partir del conocimiento que tienen del proceso los propios jóvenes con discapacidad intelectual y los profesionales implicados en los servicios y programas que intervienen en este proceso de transición, tanto en el ámbito educativo escolar como en el postescolar. Entre los dispositivos del ámbito escolar están las escuelas ordinarias y especiales, los institutos de educación secundaria, los equipos multiprofesionales de apoyo y los programas específicos que ofrece el sistema educativo. En cuanto a los 
servicios postescolares, encontramos centros ocupacionales, servicios de empleo con apoyo, programas de formación ocupacional, etc.

Con el objetivo de garantizar la confiabilidad de los resultados de la investigación se obtienen datos de diferentes fuentes; concretamente, han participado en la investigación profesionales que desarrollan su rol laboral en servicios socioeducativos relacionados con la transición, expertos en este ámbito y personas con discapacidad intelectual. También se han diseñado y aplicado diferentes instrumentos para la recogida de datos (entrevistas en profundidad y cuestionarios). Con ello se pretende disponer de un amplio abanico de datos que permitan contrastar la información mediante la triangulación de datos en las distintas etapas.

El estudio se ha llevado a cabo en tres etapas y en cada una de ellas se ha diseñado y aplicado un instrumento específico. El propósito de la primera etapa es conocer la visión que tienen los profesionales de los distintos dispositivos, sean escolares o postescolares, sobre los procesos de transición a la edad adulta y a la vida activa de los jóvenes con discapacidad intelectual. La segunda etapa busca conocer la visión de la transición que tienen personas con discapacidad intelectual que se hallan en distintas fases de su proceso de transición. En ambas etapas se diseñan y aplican entrevistas individuales.

Finalmente, la tercera fase del estudio tiene como objetivo obtener datos sobre la adecuación de los servicios o dispositivos donde se trabaja con jóvenes con discapacidad intelectual a lo largo de su proceso de transición a la edad adulta y vida activa, tanto en el escenario escolar como en el postescolar, y detectar qué obstáculos y dificultades son percibidos por parte de los expertos en estos escenarios en relación con ciertos temas que la investigación existente ha mostrado como cuestiones clave en el proceso de tránsito a la edad adulta y vida activa que siguen estos jóvenes. Para llevar a cabo este estudio seguimos el método Delphi, cuyo objetivo es la obtención de una opinión grupal fidedigna a partir de un conjunto de expertos (Landeta, I999). Es un método apropiado cuando los investigadores están interesados en recoger los juicios de expertos sobre un tema particular para documentar y valorar estos juicios, captar áreas de conocimiento colectivo sostenido por profesionales que normalmente no son verbalizadas ni exploradas y para estimular que surjan nuevas ideas relacionadas con un tema (Franklin y Hart, 2007; Martínez Piñeiro, 2003). El método Delphi comprende varias etapas sucesivas de envíos de cuestionarios, de análisis e interpretación de los datos. En cada ronda, cada experto expresa su opinión sobre un tema y recibe feedback sobre el conjunto de opiniones expresadas por el grupo. De este modo, cada persona puede saber hasta qué punto coincide o se aparta de la opinión del grupo y puede argumentar sus respuestas (Ruíz Olabuénaga y Ispízua, I989). Concretamente, en nuestro estudio se han constituido dos paneles de expertos (ámbito escolar y postescolar) y se han llevado a cabo dos rondas de cuestionarios para cada uno de los paneles de expertos (Pallisera et al., 2013).

La Tabla I resume la información sobre los objetivos de cada una de las etapas, los participantes y los temas planteados en cada etapa.

\section{Análisis de la información}

El procedimiento seguido para el análisis de datos ha sido el siguiente: en primer lugar, se ha realizado un análisis de los datos obtenidos en cada una de las etapas (ver procedimiento en Tabla 2). Posteriormente, en una segunda fase, se realiza el análisis conjunto de la información obtenida.

Una vez ha finalizado el análisis de datos obtenido en las tres etapas de la investigación, se ha realizado una segunda fase de análisis. En esta segunda fase se ha llevado a cabo la lectura en profundidad de los informes resultantes de cada una de las etapas del estudio. Se han revisado también, para profundizar en la información obtenida a partir de cada 
Tabla 1. Participantes, finalidad, instrumento y temas de trabajo en cada una de las etapas del estudio

\begin{tabular}{|c|c|c|c|}
\hline $\begin{array}{c}\text { Etapa del } \\
\text { estudio }\end{array}$ & Finalidad de la etapa & Participantes y Descripción & Temas \\
\hline 1 & $\begin{array}{l}\text { Conocer la visión de } \\
\text { los profesionales de } \\
\text { dispositivos escolares y } \\
\text { postescolares sobre la } \\
\text { transición }\end{array}$ & $\begin{array}{l}45 \text { profesionales de servicios escolares } \\
\text { y postescolares (Cataluña) } \\
80 \% \text { mujeres y } 20 \% \text { varones. El } 60 \% \\
\text { trabaja en el ámbito escolar ( } 8 \text { en } \\
\text { centros de educación especial, } 9 \text { en } \\
\text { centros ordinarios y } 10 \text { en equipos } \\
\text { multiprofesionales). El } 40 \% \text { en } \\
\text { servicios postescolares ( } 6 \text { en Centros } \\
\text { Ocupacionales, } 7 \text { en servicios de Empleo } \\
\text { con Apoyo, } 3 \text { en formación ocupacional y } \\
2 \text { en apoyos a la vida independiente). } \\
\text { El } 64 \% \text { de los participantes tiene más de } \\
15 \text { años de experiencia, el } 33 \% \text { entre } 8 \text { y } \\
15 \text { años. }\end{array}$ & $\begin{array}{l}\text { - Visión del proceso de transición } \\
\text { de jóvenes con discapacidad } \\
\text { intelectual (JDI) } \\
\text { - Visión deseada del proceso de } \\
\text { transición JDI. Propuestas de } \\
\text { cambio para la mejora: } \\
\text { - A nivel organizativo y curricular } \\
\text { - A nivel profesional } \\
\text { - A nivel de articulación/ } \\
\text { coordinación de servicios }\end{array}$ \\
\hline 2 & $\begin{array}{l}\text { Conocer la visión } \\
\text { de la transición que } \\
\text { tienen personas con } \\
\text { discapacidad que se } \\
\text { encuentran en distintos } \\
\text { momentos del proceso de } \\
\text { tránsito }\end{array}$ & $\begin{array}{l}20 \text { personas con discapacidad } \\
\text { intelectual (Cataluña) } \\
50 \% \text { mujeres y } 50 \% \text { varones. } \\
9 \text { personas tienen de } 17 \text { a } 25 \text { años, } 6 \text { de } \\
26 \text { a } 35 \text { años y } 5 \text { son mayores de } 35 . \\
7 \text { de los participantes realizan un curso } \\
\text { de formación para la transición y viven } \\
\text { en casa; una persona está en un centro } \\
\text { ocupacional y el resto trabaja en centros } \\
\text { especiales de empleo o mediante empleo } \\
\text { con apoyo. }\end{array}$ & $\begin{array}{l}\text { Visión sobre la formación previa y } \\
\text { su percepción sobre los apoyos } \\
\text { recibidos en la etapa de transición } \\
\text { - Experiencia laboral y apoyos } \\
\text { recibidos } \\
\text { - Proyecto de futuro y percepción } \\
\text { de apoyos que pueden ayudarles a } \\
\text { conseguirlos }\end{array}$ \\
\hline 3 & $\begin{array}{l}\text { Obtener datos sobre } \\
\text { la adecuación de los } \\
\text { servicios o dispositivos } \\
\text { donde se trabaja con } \\
\text { jóvenes con discapacidad } \\
\text { intelectual en el proceso } \\
\text { de tránsito. }\end{array}$ & $\begin{array}{l}2 \text { Paneles de expertos, uno de ámbito } \\
\text { escolar y otro postescolar } \\
\text { Ámbito escolar: } 20 \text { personas ( } 6 \\
\text { profesionales trabajan en centros } \\
\text { de educación especial, } 4 \text { en } \\
\text { centros ordinarios, } 4 \text { en equipos } \\
\text { multiprofesionales, } 3 \text { en programas } \\
\text { de diversificación curricular y } 3 \text { son } \\
\text { investigadores). } \\
\text { Ámbito postescolar: } 17 \text { personas (5 en } \\
\text { centros ocupacionales, } 5 \text { en empleo con } \\
\text { apoyo, } 2 \text { en apoyos a vida independiente, } \\
2 \text { en formación ocupacional y } 3 \\
\text { investigadores). } \\
\\
\text { El } 75 \% \text { son mujeres, el } 70 \% \text { tiene más de } \\
15 \text { años de experiencia y el } 30 \% \text { entre } 8 \\
\text { y } 15 \text { años. } \\
\text { Los participantes dedicados a la } \\
\text { investigación son expertos universitarios } \\
\text { en la temática procedentes de distintas } \\
\text { universidades de España. }\end{array}$ & $\begin{array}{l}\text { - Visión del proceso de transición } \\
\text { - Establecimiento sistematizado de } \\
\text { itinerarios de apoyo } \\
\text { - Trabajo con familias } \\
\text { - Adecuación de la formación de los } \\
\text { profesionales } \\
\text { - Aspectos curriculares y } \\
\text { organizativos } \\
\text { - Trabajo colaborativo entre } \\
\text { profesionales } \\
\text { - Adecuación de las alternativas } \\
\text { postescolares } \\
\text { - Coordinación de servicios }\end{array}$ \\
\hline
\end{tabular}

Fuente: Elaboración propia. 
Tabla 2. Primera fase de análisis: aplicación y procedimiento de análisis de los datos en cada uno de los estudios

\begin{tabular}{|c|c|}
\hline Etapa/instrumento & Procedimiento de aplicación y análisis de los datos \\
\hline $\begin{array}{l}\text { Etapa 1: Entrevistas } \\
\text { en profundidad a } \\
\text { profesionales (marzo- } \\
\text { abril 2012) }\end{array}$ & $\begin{array}{l}\text { Los participantes dan su consentimiento informado para la grabación de las } \\
\text { entrevistas. Se aplican las entrevistas en el puesto de trabajo de cada uno de los } \\
\text { entrevistados. } \\
\text { Se transcriben y analizan las entrevistas con el programa Atlas-ti. Se realiza un } \\
\text { análisis de contenido temático clasificando la información en función de los ejes } \\
\text { que articulan la entrevista. } \\
\text { Se redacta un informe con la información resultante del análisis. Este informe } \\
\text { comprende el análisis de las tendencias en cada uno de los códigos establecidos } \\
\text { junto con fragmentos de las entrevistas que dan apoyo a esta información. }\end{array}$ \\
\hline $\begin{array}{l}\text { Etapa 2: Entrevistas en } \\
\text { profundidad a jóvenes } \\
\text { con discapacidad } \\
\text { intelectual (abril-julio } \\
\text { 2012) }\end{array}$ & $\begin{array}{l}\text { Los participantes dan su consentimiento informado para la grabación de las } \\
\text { entrevistas. Se realizan las entrevistas de forma individual. } \\
\text { Se transcriben las entrevistas. Se redactan las trayectorias individuales a partir de } \\
\text { las experiencias narradas, con lo que se obtienen las } 20 \text { trayectorias biográficas } \\
\text { correspondientes a cada caso. } \\
\text { Se analizan las transcripciones con el programa Atlas-ti. Se realiza un análisis de } \\
\text { contenido temático conjunto clasificando la información a partir de los ejes que } \\
\text { articulan la entrevista. Ello permite analizar las tendencias en los itinerarios vividos } \\
\text { en los distintos ejes temáticos contemplados en los instrumentos. } \\
\text { Se redacta un informe final con la información obtenida sobre las trayectorias } \\
\text { personales vividas por los jóvenes con discapacidad analizada a partir de este } \\
\text { proceso. }\end{array}$ \\
\hline $\begin{array}{l}\text { Etapa 3: Cuestionarios a } \\
\text { profesionales siguiendo } \\
\text { el método Delphi } \\
\text { (marzo-junio 2012) }\end{array}$ & $\begin{array}{l}1^{a} \text { ronda cuestionarios: Se pide a cada participante que valore cada ítem en una } \\
\text { escala del } 1 \text { al } 5 \text { en función de su grado de acuerdo con la afirmación presentada. } \\
\text { Se les invita a escribir, para cada ítem, sus comentarios, dudas, apreciaciones } \\
\text { sobre la afirmación en cuestión para argumentar su respuesta. } \\
\text { Los cuestionarios se envían por correo electrónico y los participantes realizan la } \\
\text { devolución por esta vía una vez cumplimentados. Se analizan separadamente } \\
\text { los cuestionarios de cada panel de expertos. Se calculan medias y desviaciones } \\
\text { típicas de cada ítem y los coeficientes de variación como indicador del grado de } \\
\text { consenso entre los diferentes expertos. Se realiza un análisis de contenido de las } \\
\text { repuestas abiertas. } \\
2^{a} \text { ronda de cuestionarios: Dado que los expertos no sugieren cuestiones distintas } \\
\text { a las planteadas en el primer cuestionario, se envía el mismo cuestionario a los } \\
34 \text { expertos que respondieron el primero. A cada miembro se le envía también } \\
\text { el cuestionario con las puntuaciones individuales de cada experto en la primera } \\
\text { vuelta, media y desviación típica, y el resumen del análisis de los comentarios de } \\
\text { los expertos. } \\
\text { Para analizar el segundo cuestionario se sigue el mismo procedimiento que en la } \\
\text { primera ronda. } \\
\text { El análisis de datos se articula en función de los ejes temáticos del cuestionario. } \\
\text { Se redacta un informe final en el que se recogen los resultados obtenidos, con las } \\
\text { tendencias resultantes y fragmentos de los textos escritos por los participantes en } \\
\text { su respuesta a las preguntas abiertas }{ }^{*} \text {. }\end{array}$ \\
\hline
\end{tabular}

("El procedimiento seguido y los resultados obtenidos en el estudio realizado a partir del método Delphi pueden consultarse en Pallisera et al. (2OI3).

Fuente: Elaboración propia. 
uno de los instrumentos, las transcripciones de las entrevistas a los profesionales y a las personas con discapacidad y de las opiniones mostradas por los expertos participantes en el estudio Delphi. En base a todo este proceso se definen los ejes temáticos que van a centrar el análisis conjunto de la información obtenida. En esta segunda fase nos interesa obtener una comprensión global acerca de cómo se produce el proceso de tránsito a través de la red de dispositivos o servicios escolares y postescolares que ofrecen apoyos a los jóvenes con discapacidad en sus trayectorias hacia la vida adulta. Se definen, concretamente, los siguientes ejes temáticos de análisis:

- Adecuación de los dispositivos (escolares y postescolares) en relación con el proceso de tránsito: funciones, responsabilidades, coordinación de servicios y apoyos.

- La formación para la transición: la formación de los jóvenes para el logro de objetivos relacionados con la inclusión social y laboral, para la emancipación familiar y la participación comunitaria.

- El papel de los jóvenes y sus familias en el proceso de tránsito: oportunidades de participación.

Disponer de información procedente de distintas fuentes (profesionales y jóvenes) y obtenida con distintos instrumentos (entrevistas en profundidad y técnica Delphi) ha facilitado realizar un proceso de triangulación de la información, a partir de la cual se definen las cuestiones prioritarias en los distintos temas (Cisterna, 2005). Concretamente, se usa la triangulación de métodos, de datos y de investigadores (Okuda y Gómez, 2005). La triangulación de métodos se ha llevado a cabo con el uso de distintos acercamientos al fenómeno estudiado: concretamente, se han realizado las entrevistas a profesionales (etapa I); entrevistas a las personas con discapacidad, en base a las cuales se han redactado las trayectorias personales individuales (etapa 2); se ha aplicado el método Delphi, que supone una aproximación mixta (cuantitativacualitativa) en la tercera etapa. La triangulación de datos se produce a través de la verificación y comparación de la información obtenida en diferentes momentos mediante los distintos métodos utilizados. También se utiliza la triangulación de investigadores, puesto que los miembros del equipo participan en el análisis de los datos; en algunos momentos de forma independiente y otras a través del trabajo en equipo, y finalmente se llega a un consenso entre ellos.

\section{Resultados}

El análisis desarrollado nos permite disponer de una visión contrastada y amplia del proceso de tránsito a la vida adulta de los jóvenes con discapacidad. A continuación presentaremos los principales resultados derivados de esta investigación. En primer lugar, se plantean distintas cuestiones relacionadas con los dispositivos y sus relaciones; concretamente: la continuidad de los apoyos que se ofrecen a los jóvenes con discapacidad en el transcurso del proceso de tránsito, la adecuación de los servicios o dispositivos, y la relación entre estos dispositivos. En segundo lugar, se aborda el tema de la formación que reciben los jóvenes como preparación para la transición a su vida adulta y el logro de los objetivos asociados a ella: inclusión laboral y social, participación comunitaria, etc. Finalmente, nos referimos a un tema clave en los procesos de tránsito: la participación de los jóvenes y sus familias en el proceso.

En la presentación de la información de cada uno de estos temas nos centramos específicamente en las cuestiones que, a la luz del análisis realizado, se configuran como elementos clave a tener en cuenta para mejorar los procesos de tránsito de los jóvenes con discapacidad en nuestro país. Los datos se han obtenido a partir del proceso de análisis descrito en el apartado anterior. En algunos casos, se ilustra la información señalada con fragmentos extraídos de las entrevistas o de los cuestionarios Delphi. 


\subsection{Los dispositivos que integran la red de servicios de tránsito para jóvenes con discapacidad intelectual: servicios escolares y postescolares}

Las trayectorias de los jóvenes con discapacidad en su proceso de tránsito a la vida adulta están enormemente condicionadas por el eje espacio/ tiempo. Así, el estudio desarrollado en la etapa 2 nos ha permitido obtener 20 trayectorias de vida diversificadas en función de distintas variables, pero fuertemente influenciadas por las tendencias en escolarización y atención a las personas con discapacidad mayoritarias en el momento en que estas personas fueron escolarizadas.

Podemos establecer dos situaciones dominantes: por una parte, las personas que se escolarizaron en las décadas 70 y 80 , que asistieron a centros de educación especial y sus itinerarios postescolares transcurrieron básicamente en centros de atención a personas adultas con discapacidad (centros ocupacionales o centros especiales de empleo); por otra, la de personas que han sido escolarizadas a partir de la década de los 9o. En este último caso, los itinerarios escolares son diversificados.

El progresivo avance de la inclusión educativa vivido en el Estado español a partir de la Ley de Ordenación General del Sistema Educativo (LOGSE, I990) y la Ley de Educación (LOE, 2006) conlleva el aumento de trayectorias de personas que inician y desarrollan su escolarización en centros ordinarios. Se percibe en las personas más jóvenes una mayor diversificación en los itinerarios postescolares: algunas de ellas continúan asistiendo a centros ocupacionales o centros especiales de empleo, pero otras acceden a otras alternativas de carácter inclusivo como los servicios de empleo con apoyo. También se percibe un aumento, en los últimos años, de personas que después de finalizar la escolarización obligatoria han realizado estudios de formación profesional. Aunque algunas de estas experiencias no han sido satisfactorias, son una muestra del incremento de posibilidades existentes para los jóvenes con discapacidad en su transición a la vida adulta. Sin duda, como plantean Kaehne y Beyer (2009), la apuesta por la educación inclusiva genera nuevos retos para la transición, puesto que la incorporación de centros educativos ordinarios en la red de servicios de transición obliga al establecimiento de alternativas que den continuidad a la educación inclusiva que han recibido en los últimos años muchos jóvenes con discapacidad intelectual.

En los momentos actuales la red de dispositivos o servicios de transición en nuestro país es diversificada y compleja. Conocer cómo se produce el proceso de tránsito implica profundizar en la red de dispositivos. En este sentido, se analizan seguidamente distintos temas que los participantes en el estudio han destacado sobre la relación entre los centros o dispositivos donde se atiende a jóvenes con discapacidad en su proceso de tránsito a la vida adulta:

- La articulación de los apoyos que reciben a lo largo del proceso.

- La adecuación de los servicios o dispositivos.

- La distribución de responsabilidades y funciones entre los servicios o dispositivos.

\section{a. La articulación de los apoyos que se ofrecen a los jóvenes con discapacidad en el transcurso del proceso de tránsito}

Un elemento característico del proceso de transición a la vida adulta que viven los jóvenes con discapacidad es que a lo largo de su trayectoria se valora para cada joven los apoyos que necesita y como deben ofrecerse en los distintos centros o dispositivos por donde transcurre su itinerario (Foley et al., 20I2). En este sentido, nos interesa analizar si existe continuidad o fragmentación en las trayectorias que establecen los jóvenes con discapacidad a lo largo del proceso de tránsito.

En primer lugar, se aprecia que en la transición de primaria a secundaria los jóvenes con discapacidad intelectual tienden a ser atendidos en programas de diversificación curricular, 
pasando buena parte de la jornada escolar en aulas de apoyo y con adaptaciones curriculares:

«Los exámenes un poco especiales porque claro, yo, yo hay cosas que a mi si m dicen, "bum, bum, bum" no me entero. Si me lo explican pausado y eso, sí. Ahora, si van del tirón, yo no me entero de nada. Y bueno es un poco (...) bueno, los exámenes eran un poco diferentes. O sea, era lo mismo pero con preguntas más sencillas. Y bueno, gracias a los exámenes aprobé, no todos, pero si algunos» (Persona con discapacidad, entrevista 7).

Esta situación, aunque se reconoce que puede ayudar en el proceso de aprendizaje, es discutida por algunas de las propias personas con discapacidad:

«Es que yo los grupos creo que tienen que ser heterogéneos (...) Que después de la escuela hagan una hora (...) porque si no creo que de alguna manera se sentirán rechazados, que se sentirán como los jóvenes que van a una escuela especial» (Persona con discapacidad, entrevista Iо).

Así, tanto los profesionales entrevistados como las personas que han integrado los dos paneles de expertos y las propias personas con discapacidad entrevistadas coinciden en señalar abiertamente que los jóvenes con discapacidad intelectual experimentan dificultades para completar los estudios de secundaria obligatoria. Para los jóvenes que no lo han conseguido, esto constituye una percepción de fracaso personal puesto que les limita las oportunidades de acceso a la formación y al empleo:
«Desgraciadamente no pude obtener el graduado, por eso estoy aquí (un programa específico para jóvenes con discapacidad intelectual). Mira que lo intenté y puse ganas, pero al final no pude (...) Y si pudiera volver atrás y sacarme el graduado, lo haría» (Persona con discapacidad, entrevista 5).

Incluso en el caso de que puedan acreditar esta formación, difícilmente pueden continuar su formación más allá de la Enseñanza Secundaria
Obligatoria. Aunque algunas personas lo intentan, se trata de un objetivo realmente complejo. En la etapa de formación profesional hay poca experiencia en la realización de adaptaciones curriculares por lo que superar los cursos se convierte en una tarea difícil:

«Yo cuando acabé la Educación Secundaria Obligatoria, fui al ciclo formativo de auxiliar sanitario, y el primer día me dijeron que no aprobaría, porqué había mucha materia y era muy difícil. Tuve que cambiar y pasarme a hostelería» (Persona con discapacidad, entrevista II).

La alternativa mayoritaria, cuando finalizan la escolarización, es el acceso a los centros de atención a personas adultas con discapacidad (centros ocupacionales o centros especiales de empleo) y, en menor proporción, por tratarse de alternativas menos frecuentes, los servicios de empleo con apoyo. En pocos casos acceden a los llamados "programas de segunda oportunidad" (por lo general Programas de Calificación Profesional Inicial ${ }^{2}$ ), que en la mayoría de los casos tienen carácter específico y se ofrecen, por lo general, por parte de organizaciones del ámbito de la discapacidad. Los profesionales están de acuerdo con que deberían habilitarse vías de acceso y de apoyo claramente estructuradas en la formación profesional ${ }^{3}$ para posibilitar la formación para el empleo de los jóvenes:

«Deberíamos estudiar de los ciclos formativos de grado medio de que disponemos, cuáles

2. Los Programas de Calificación Profesional Inicial son programas de formación profesional inicial a los que pueden acceder jóvenes que no han obtenido el título de Graduado en la enseñanza secundaria obligatoria. Preparan para el desempeño de una profesión, al mismo tiempo que profundizan en la formación básica.

3. La formación profesional se organiza en cursos de grado medio y de grado superior. Para acceder al grado medio, debe superarse la Educación Secundaria Obligatoria o bien, en caso de no disponer del Graduado, debe superarse una prueba de acceso o un curso de acceso específico y tener I 7 años. Para acceder al nivel superior, debe disponerse del Bachillerato o, en caso de que no se haya cursado, superar una prueba de acceso y tener I9 años. Los alumnos con formación profesional de grado medio pueden acceder al superior superando una prueba específica. Estos requisitos hacen que, en nuestro contexto, pocas personas con discapacidad intelectual puedan acceder a la formación profesional reglada. 
serían susceptibles de ser adaptados para favorecer que estos jóvenes pudieran acceder a una formación profesional» (Profesional, entrevista I2).

En relación a la temática, profesionales y personas con discapacidad están de acuerdo con la necesidad de diversificar las opciones formativas. Existen, en estos momentos, pocas opciones y centradas en pocos ámbitos profesionales:

«Se tienen que buscar alternativas, pero no en un solo ámbito sino en muchos más. Yo tuve que cursar la formación de carpintería, que no me interesaba nada, porque era la única opción que había en el centro» (Persona con discapacidad, entrevista Io).

\section{b. La adecuación de los servicios o dispositivos}

Aunque los dispositivos donde reciben atención mayoritaria los jóvenes con discapacidad intelectual cuando finalizan su etapa educativa tienen sin duda una función imprescindible en la atención a personas adultas con discapacidad intelectual, puede considerarse que, en general, estos recursos no son los más adecuados para los jóvenes cuando finalizan su escolarización. Básicamente, por dos razones: en primer lugar, porque se trata espacios donde sólo se trabaja con personas con discapacidad (planteando en este sentido una ruptura con la inclusión educativa que han experimentado muchos jóvenes); en segundo lugar, porque estos centros se orientan básicamente a dar respuesta a las necesidades que experimentan las personas adultas. Es en este sentido que algunos de los participantes en la investigación, especialmente los que trabajan en el diseño y aplicación de apoyos en centros educativos ordinarios, consideran que los servicios postescolares no son del todo adecuados. Ello no quiere decir que se deban organizar nuevos servicios, distintos a los existentes, sino implementar las vías para que los jóvenes con discapacidad intelectual puedan participar de los dispositivos ya existentes para el resto de los jóvenes, sea posibilitando su continuidad en el sistema educativo, sea participando en los programas que se establecen para potenciar la transición a la vida activa (Programas de Calificación Profesional Inicial, formación ocupacional, etc.). Así lo plantea uno de los profesionales que integró un panel de expertos en el estudio Delphi:

«Podemos cuestionar si los servicios postescolares son adecuados. Se trata en su mayoría de espacios específicos para personas con discapacidad. Sería favorable diseñar procesos accesibles reales utilizando los entornos que ya existen en los procesos de transición del resto de jóvenes» (Profesional, Delphi I2).

\section{c. La distribución de responsabilidades y funciones entre los servicios o dispositivos relacionados con el proceso de tránsito}

La coordinación entre los diversos dispositivos implicados en el proceso de transición, en la etapa escolar y la postescolar, es un elemento clave para asegurar que los jóvenes puedan construir trayectorias de transición que les permitan conseguir los objetivos relacionados con la vida adulta (Barron y Hassiotis, 2008; Beyer et al., 2008). En este sentido, es importante la existencia de un buen liderazgo para potenciar la transición (Hudson, 2006). De los estudios sobre el tema se desprende la recomendación de que se establezcan a nivel político-administrativo directrices orientadoras de los procesos de tránsito (Beyer y Kaehne, 2008; Rush et al., 2009). También se apunta el papel estratégico que desempeña el ámbito territorial de proximidad en todo el proceso y especialmente en algunas etapas del mismo, por ejemplo, para facilitar las prácticas laborales en procesos de formación profesional o inclusión laboral (Kochhar-Bryant y Greene, 2009).

En nuestro país el período de transición no tiene una consideración específica a nivel normativo; tampoco se ha establecido, a nivel de la administración educativa, un procedimiento que clarifique las acciones a realizar por parte de los servicios implicados, los estadios o etapas que siguen los jóvenes en este proceso, el rol que 
juega cada organización en cada etapa, el tipo de información que compartirán sobre los jóvenes que están realizando el proceso y la provisión de información sobre las opciones futuras. Ello facilitaría la participación activa en la toma de decisiones de los auténticos protagonistas del proceso, los jóvenes y sus familias.

$\mathrm{Al}$ no existir un proceso claramente establecido, las decisiones sobre las etapas a seguir, los contactos a establecer con servicios postescolares, y las reuniones a realizar tanto con los jóvenes y sus familias como con los servicios postescolares, quedan a decisión en la mayoría de los casos de los profesionales del centro educativo. Se echa en falta, también por este motivo, la existencia de un claro liderazgo que debería recaer en los agentes educativos, puesto que es en el escenario educativo donde el joven inicia su proceso de transición y desde donde se toman las decisiones que van a condicionar su trayectoria. En algunos ámbitos territoriales, siempre en función de iniciativas particulares de los servicios que en él se ubican, se han establecido algunos procedimientos con el objeto de sistematizar los itinerarios de transición; sin embargo, se trata, en estos momentos, de planteamientos minoritarios. Los profesionales entrevistados, y también los expertos participantes en el estudio Delphi, se han mostrado partidarios de potenciar la coordinación entre servicios en base a la articulación territorial, como estrategia para favorecer el establecimiento de apoyos personalizados que tengan en cuenta las perspectivas de los distintos agentes implicados:

«Creo que el gobierno debería llevar la iniciativa y organizar a nivel territorial unas comisiones de coordinación con representantes de los distintos departamentos (educación, servicios sociales, salud, etc.), y aquí estarían también miembros de los equipos multiprofesionales, representantes de las familias, los ayuntamientos, y la comisión debería tener como función la planificación, gestión y evaluación de las actuaciones en la transición (...)» (Profesional, entrevista I 5 ).
4.2. La formación para la transición: la formación para el logro de objetivos relacionados con la inclusión social y laboral, para la emancipación familiar y la participación comunitaria

Sin duda, la formación recibida por los jóvenes con discapacidad es un elemento clave para facilitar su posterior inclusión laboral y social. Concretamente, diferentes estudios plantean la relación entre las habilidades relacionadas con la autodeterminación (toma de decisiones, resolución de problemas, habilidades para establecer objetivos, asumir riesgos, autodefensa, autoconocimiento) y la inclusión sociolaboral (Madaus et al., 2008; Test et al., 2009; Wehmeyer y Palmer, 2003). Asumir estos planteamientos tiene consecuencias en la configuración de los currículos formativos, que deberían potenciar estas habilidades (Izuzquiza, 2002; Pallisera, 20I Ib; Vilà et al., 20I2).

El tema de la formación que reciben los jóvenes con discapacidad intelectual para el logro de objetivos relacionados con la inclusión laboral y social se ha indagado fundamentalmente a través del cuestionario Delphi, aunque también se ha obtenido información sobre esta cuestión a partir de las entrevistas realizadas a profesionales y a las personas con discapacidad.

En general, los profesionales muestran consenso en plantear que, en nuestro contexto, en los institutos de secundaria apenas se trabajan estos contenidos. Dos son los principales motivos a los que aluden los profesionales: en primer lugar, se plantea que al no tener la transición a la edad adulta una consideración específica en las políticas educativas y sociales de nuestro país, no existen unas orientaciones curriculares en base a las cuales organizar los apoyos para los jóvenes con discapacidad intelectual:

«Se echa en falta un modelo curricular que establezca las directrices para el período de transición, que ayude a concretar, desde el centro educativo, los apoyos curriculares necesarios y personalizados para cada alumno» (Profesional, entrevista ıо). 
Con ello, es cada centro y, más concretamente, los profesionales responsables de la acción psicopedagógica y orientadora, quienes toman decisiones sobre las adaptaciones curriculares que se diseñan y aplican al alumnado con discapacidad.

En segundo lugar, los profesionales comentan que los contenidos disciplinares tienen un peso específico considerable en el currículo de secundaria, por lo que las adaptaciones curriculares que se realizan con los jóvenes con discapacidad intelectual se centran, por lo general, en el ámbito académico:

$$
\begin{aligned}
& \text { «A pesar de que a nivel teórico estamos } \\
& \text { centrados en el desarrollo de competencias } \\
& \text { como la autodeterminación, a nivel práctico, y } \\
& \text { según mi experiencia como asesora a centros, } \\
& \text { aún estamos centrados en un paradigma de } \\
& \text { acumulación de contenidos» (Profesional, } \\
& \text { Delphi 7). }
\end{aligned}
$$

Las personas con discapacidad que han estado escolarizadas en centros ordinarios valoran de distinta manera los aprendizajes realizados, en función de sus experiencias particulares de escolarización. Así, un participante valora positivamente la formación recibida, concretamente la relacionada con el uso del dinero:

«En mates tocas también el dinero. Me han servido mucho para el manejo del dinero las mates» (Persona con discapacidad, entrevista $\mathrm{I} 7$ ).

Mientras que otro realiza una valoración no tan positiva:

«A mí el instituto me han ayudado pero no tanto. O sea, no nos han hablado de vida adulta y todo esto» (Persona con discapacidad, entrevista 2).

Sin embargo, parece que en los centros de educación especial la situación es sensiblemente distinta, puesto que sus profesionales consideran que en ellos no existe de forma tan evidente la presión de los contenidos, lo que facilita poder enfatizar el desarrollo de competencias personales.

En el ámbito postescolar se considera que los contenidos relacionados con la vida adulta se trabajan con cierta intensidad, aunque algunas personas señalan la necesidad de insistir en las habilidades directamente relacionadas con la vida independiente.

\subsection{La participación de las personas con discapacidad y sus familias}

En los últimos años, la influencia del modelo social en el análisis de la discapacidad ha potenciado la incorporación de las experiencias y vivencias de las personas con discapacidad en los procesos de toma de decisiones sobre su propia vida. Durante el proceso de tránsito a la vida adulta deben tomarse decisiones que van a repercutir claramente en las oportunidades de inclusión laboral y social de los jóvenes con discapacidad. En todo el proceso es fundamental que los jóvenes y sus familias reciban la información suficiente y adecuada acerca de las alternativas de apoyo posibles; además, debería asegurarse su plena participación en el proceso de toma de decisiones. En este sentido, diversos estudios plantean que los jóvenes experimentan numerosas dificultades para participar activamente en las decisiones relacionadas con su vida futura (Beresford, 2004; Carnaby y Lewis, 2005; Cooney, 2002; Kaehne y Beyer, 2009; Lindstrom et al., 2007).

En nuestra investigación, a través de las entrevistas realizadas a las personas con discapacidad hemos podido obtener distintas visiones personales acerca de sus vivencias del proceso de tránsito y de la percepción que tienen sobre su participación en el proceso. Los procesos experimentados y las fórmulas planteadas al finalizar la escolarización han variado bastante, en función de las alternativas que ofrece el territorio, pero también de la información disponible en cada uno de los centros de secundaria por parte de los profesionales responsables de la orientación. En este sentido, la variedad de las experiencias personales son fruto, 
en gran medida, de la inexistencia de un proceso claro y sistemático que establezca los momentos clave y secuencie las decisiones a tomar en los últimos años de la escolarización.

Por parte de los profesionales, tanto en las entrevistas personales como en los cuestionarios, se incide justamente en la necesidad de un cambio profundo en el funcionamiento de los servicios, en el sentido que incluya efectivamente a los jóvenes con discapacidad en la toma de decisiones que afectan a su vida:

«Caemos en el error de tratarlos como a "sujeto pasivo", pero debemos potenciar que sean realmente sujetos activos y protagonistas reales de su vida. Debemos ser los primeros en creernos que realmente deben tener las mismas oportunidades que el resto, que deben poder escoger, tomar decisiones, etc. Y dar el apoyo necesario en cada momento de su vida» (Profesional, Delphi 3).

Varios profesionales aluden a la necesidad de incorporar decididamente sistemas de trabajo centrados en la persona y aplicarla concretamente tanto en los centros del escenario educativo como en el postescolar. Porque, en definitiva:
«Es el joven quien debe poder decir lo que le gustaría hacer, o cuál es su visión. Y debe poder decidir sobre todas las posibilidades, $y$ los profesionales debemos intentar apoyarle en su proyecto» (Profesional, entrevista 7).

Las personas con discapacidad entrevistadas coinciden en manifestar su voluntad de participar activamente en la toma de decisiones sobre sus itinerarios vitales. Para ello, insisten en la necesidad de tomar decisiones y, sobretodo, en la necesidad de creer en las posibilidades de cada uno para conseguir sus proyectos:

«Creer en uno mismo es fundamental. Creer en lo que podemos hacer, en que poniendo voluntad y esfuerzo podemos avanzar»

(Persona con discapacidad, entrevista 4).

\section{Conclusiones y propuestas}

La información obtenida a partir de la investigación desarrollada nos ha permitido una mayor comprensión del proceso de tránsito a la vida adulta de los jóvenes con discapacidad intelectual, e incidir específicamente en aquellos aspectos que deberían modificarse para mejorar las oportunidades de inclusión social y laboral de estos jóvenes. El contraste de visiones entre profesionales y expertos de los ámbitos educativo, por una parte, y postescolar, por otra, y las experiencias narradas por las propias personas con discapacidad nos llevan a plantear las siguientes estrategias de mejora:

En primer lugar, debe incorporarse la transición a la edad adulta como una etapa con entidad propia en el proceso educativo y formativo de los jóvenes con discapacidad. Esta etapa debería constituir una línea prioritaria en las políticas educativas y sociales, puesto que ambas están estrechamente relacionadas con el diseño de los itinerarios formativos y la configuración de los apoyos individuales. El avance de la inclusión educativa, a pesar de las dificultades añadidas por la crisis socioeconómica actual, genera nuevos retos a los agentes educativos y sociales implicados, puesto que se reclaman nuevas alternativas para los jóvenes, así como una transformación de los modelos de funcionamiento de los dispositivos tradicionales. Por ello es necesario que las políticas educativas otorguen a este período la trascendencia que requiere, estableciendo los planteamientos curriculares adecuados, secuenciando las acciones a desarrollar a lo largo de la educación secundaria obligatoria, proponiendo estrategias para tomar decisiones acerca de las opciones de formación postobligatorias y concretando los agentes educativos y sociales que deben ejercer responsabilidades en las distintas fases del proceso. Todo ello es necesario para que los profesionales que intervienen en la articulación de los apoyos a lo largo del proceso dispongan de las orientaciones suficientes para trabajar de forma adecuada y coordinada en los distintos centros y servicios, tanto educativos como postescolares. 
En este sentido, una línea de trabajo que estimamos necesaria es la activación del papel de los equipos multiprofesionales (los equipos de asesores y orientadores y psicopedagogos) en el liderazgo de los procesos de coordinación que deban establecerse en la organización del tránsito a la vida adulta de los jóvenes con discapacidad. El conocimiento que disponen del territorio y de su oferta educativa y formativa, así como su experiencia en los procesos de asesoramiento educativo les coloca en una posición idónea para jugar un papel clave en el proceso de tránsito y articular colaborativamente la actuación de los distintos implicados en el territorio. Pero, para ello, nuevamente, debe reclamarse que las políticas educativas otorguen al período de transición la consideración que demanda como período clave en la configuración de trayectorias personales inclusivas.

Otra línea de trabajo fundamental es otorgar a los jóvenes con discapacidad el protagonismo que merecen como centro de los procesos de tránsito; facilitar su plena participación en los procesos dirigidos a la toma de decisiones relacionadas con su trayectoria. Y posibilitar, sobre todo, los apoyos que puedan permitir al joven construir su proyecto personal de formación. Ello implica no sólo tener en cuenta su participación en la planificación de la transición, sino también revisar en profundidad el funcionamiento actual de los servicios educativos y sociales para asegurar que las personas constituyan efectivamente el centro de su acción.

La transición a la vida adulta de los jóvenes con discapacidad debe compartir sus objetivos con la transición del resto de los jóvenes. Por ello, trabajar para mejorarla en el sentido que hemos planteado en este artículo debería contribuir, en general, a la madurez socioeducativa de nuestro sistema. 
Adame, M.T. y Salvà, F. (20I0): “Abandono escolar prematuro y transición a la vida activa en una economía turística: el caso de Baleares", Revista de Educación, 35 I: I85-210.

Barron, A. \& Hassiotis, A. (2008): "Good practice in transition services for Young people with LD: a review", Advances in Mental Health and Learning Disabilities, 2: 18-23.

Becerra, M. T. et al. (2012): "Programas de orientación y empleo con apoyos de personas con discapacidad intelectual. Modelos y estrategias de intervención”, Revista Española de Orientación y Psicopedagogía, 23(3): 9-I 5.

Beresford, B. (2004): "On the road to nowhere? Young disabled people and transition", Child: Care, Health \& Development, $30(6): 58$ I-587.

Beyer, S. \& Kaehne, A. (2008): “The transition of young people with learning disabilities to employment: What works?”, Journal on Developmental Disabilities, I4(I): 85-94.

Beyer, S. et al. (2008): What works? Transition to employment for young people with learning disabilities, Cardiff: Welsh Centre for Learning Disabilities.

Carnaby, S. \& Lewis, P. (2005): “Involving young people with Learning Disabilities Leaving School en Planning for the Future". En: Cambridge y Carnaby (eds.): Person Centred Planning and Care Management wih Person with Learnig Disabilities, London: Jessica Kingsley: 84-99.

Castro, M. (2013): "Els Programes de Qualificació Professional Inicial: Un entorn formatiu inclusiu per a Persones amb Discapacitat”. In: II Cimie.

Castro, M. y Vilà, M. (20I3): "La inclusión de personas con discapacidad en Planes de Transición al Trabajo”, Revista Española de Orientación y Psicopedagogía, 24: IOO-I I 5.

Cisterna, F. (2005): “Categorización y triangulación como procesos de validación del conocimiento en investigación cualitativa”, Theoria, I4(I): 6I-7I.

Cobb, R. B. \& Alwell, M. (2009): “Transition Planning. Coordinating Interventions for Youth with Disabilities", Career Developmental for Exceptional Individuals, 32(2): 70-8I.

Cooney, B. F. (2002): "Exploring Perspectives on Transition of Youth With Disabilities: Voices of Young Adults, Parents, and Professionals”, Mental Retardation, 40(6): 425-435.

Egido, I. et al. (2009): "La inclusión social y laboral de las personas con discapacidad intelectual mediante los programas de empleo con apoyo. Un reto para la orientación”, Revista Española de Orientación y Psicopedagogía, 20(2): I35-46.

España. Ley Orgánica 2/2006, de 3 de mayo, de Educación, Boletín Oficial del Estado, núm. I06, de 4 de mayo de 2006, pp. I7I 58-I7207.

España. Ley Orgánica I/ı990 de 3 de octubre, de Ordenación General del Sistema Educativo, Boletín Oficial del Estado, núm. 238, de 4 de octubre de I990, pp. 28927-28942.

Fernández, S. y Iglesias, M. T. (2005): “La transición escuela-empleo de las personas con discapacidad en España: una investigación institucional mediante estudio de casos", Bordón, 57(4): 507-518.

Foley, K-R. et al. (20I2): "Young adults with intelellectual disability transitioning from school to post-school: A literature review framed within the ICF", Disability o Rehabilitation, 34(20): I747-I764.

Franklin, K. K. \& Hart, J. K. (2007): "Idea Generation and Exploration Benefits and Limitations of the Policy Delphi Research Method", Innovative Higher Education, $3 \mathrm{I}$ : 237-246.

Gill, C. J. (I999): “Invisible Ubiquity: The surprising relevance of Disability Issues in Evaluation", American Journal of Evaluation, 20(2): 279-297. 
Hudson, B. (2006): “Making and missing connections: learning disability services and the transition from adolescence to adulthood", Disability \& Society, 2I(I): 47-60.

Izuzquiza, D. (2002): "La autodeterminación en el currículo de la acción tutorial para alumnos con Discapacidad Intelectual. Propuestas didácticas para la escuela inclusiva”, Tendencias Pedagógicas, 7: 247-259.

Jordán de Urríes, F. B. et al. (2005): Análisis de la evolución del Empleo con Apoyo en España, Madrid: Real Patronato sobre Discapacidad. Documentos 59/2005.

Jordán de Urríes, F. B. y Verdugo, M.A. (20I3): "Transición a la vida adulta". En: Verdugo, M.A. y Schalock, R.L. (coord.): Discapacidad e Inclusión: Manual para la docencia, Salamanca: Amarú: 359-378.

Jordán de Urríes, F. B. \& Verdugo, M.A. (20I0): "Evaluation and follow up of Supported Employment initiatives in Spain from 1995 to 2008", Journal of Vocational Rehabilitation, 33: 39-49.

Kaehne, A. \& Beyer, S. (2009): "Views of professionals on aims and outcomes of transition for young people with learning disabilities", British Journal of Learning Disabilities, 37: I38-I44.

Kochhar-Bryant, C.A. \& Greene, G. (2009): Pathways to Successful Transition for Youth with Disabilities. A Developmental Process. $2^{\text {nd }}$ Edition, Upper Saddle River: Pearson.

Landeta, J. (1999): El método Delphi, Barcelona: Ariel.

Lindstrom, L. et al. (2007). "Voices from the Field: Recommended Transition Strategies for Students and School Staff", The Journal for Vocational Special Needs Education, 29(2): 4-II.

Madaus, J. W. et al. (2008): “Adults with Learning Disabilities in the Workforce: Lessons for Secondary Transition Programs", Learning Disabilities Research o Practice, $23(3)$ : I 48 - 53 .

Martínez Piñeiro, E. (2003): "La técnica Delphi como estrategia de consulta a los implicados en la evaluación de programas", Revista de investigación educativa, 2 I (2): 449-463.

Martinez Rueda, N. (2002): Juventud y discapacidad. Programas y herramientas para facilitar la transición a la vida adulta, Bilbao: Mensajero.

Okuda, M. y Gómez-Restrepo, C. (2005): "Métodos en investigación cualitativa: triangulación”, Revista Colombiana de Psiquiatría, 24(I): I I 8-I 24.

Olmos, P y Mas, O. (2013): “Jóvenes, fracaso escolar y programas de segunda oportunidad", Revista Española de Orientación y Psicopedagogía, 24(I): 78-93.

Pallisera, M. et al. (20I4): “ ¿Continuidad o fragmentación? Percepción de los profesionales sobre la coordinación entre servicios en los procesos de tránsito a la vida adulta de los jóvenes con discapacidad intelectual”, Educatio Siglo XXI, 32(I): 213-232.

Pallisera, M. et al. (20I3): "Transición a la vida adulta de jóvenes con discapacidad intelectual. La opinión de los profesionales de servicios escolares y postescolares", Revista Española de Orientación y Psicopedagogía, 24(2): IOO-I I 5.

Pallisera, M. et al. (2OI2): "Beyond school inclusion: secondary school and preparing for labour market inclusion for young people with disabilities in Spain", International Journal of Inclusive Education, I6(I I/I2): I I I 5-I I 29.

Pallisera, M. (20I Ia): “Transition scenarios for young people with learning disabilities in Spain. Relationships and discrepancies", European Journal of Special Needs Education, 26(4): 495-507.

Pallisera, M. (20I Ib): "La inclusión laboral y social de los jóvenes con discapacidad intelectual. El papel de la escuela”, Revista Interuniversitaria de Formación del Profesorado, 70(25/I): I 85-200.

Rius, M. (2005): Recerca sobre les persones amb discapacitat psíquica contractades a l'Administració de la Generalitat de Catalunya. Anàlisi de la incidència de la inserció laboral en diferents dimensions de la vida dels treballadors amb discapacitat psíquica. Tesis doctoral, Universitat de Girona. 
Rosselló, M. R. y Verger, S. (2008): “La inclusión de personas con discapacidad en el lugar de trabajo en las Islas Baleares", Revista Europea de Formación Profesional, 45: I8 I-200.

Ruíz Olabuénaga, J. I. y Ispizcua, M. A. (1989): La descodificación de la vida cotidiana. Métodos de investigación cualitativa, Bilbao: Universidad de Deusto.

Rush, F. R. et al. (2009): “Toward Self-Directed Learning, Post-High School School Placement, and Coordinated Support. Constructing New Transition Bridges to Adult Life", Career Development for Exceptional Individuals, 32(I): 53-59.

Test, D. W. et al. (2009): "Evidence-Based Secondary Transition Predictors for Improving Postschool Oucomes for Students With Disabilities", Career Development for Exceptional Individuals, 32(3): I60-I8I.

Trainor, A. (2008): "Using Cultural and social Capital to Improve Postsecondary Outcomes and Expand Transition Models for Youth with Disabilities", The Journal of Special Education, 42(3): I 48 - I 62.

Vilà, M. et al. (20I2): "La inclusión laboral de los jóvenes con discapacidad intelectual: un reto para la orientación psicopedagógica”, Revista
Española de Orientación y Psicopedagogía, 23(I): 85-93.

Vilà, M. et al. (20I0): “¿Contribuye a la inclusión laboral la formación que recibe el alumnado con discapacidad intelectual en la ESO? Análisis y propuestas", Revista de Educación Inclusiva, 3(3): 5 I-66.

Vilà, M. et al. (2007): "Work integration of people with disabilities in the regular labour market: What can we do to improve these processes?", Journal of Intellectual and Developmental Disability, 32(I): IO-I8.

Walmsley, J. \& Johnson, K. (2003): Inclusive Research with People with Learning Disabilities: Past, Present and Future, London: Jessica Kingsley Publishers.

Wehmeyer, M. L. \& Palmer, S. B. (2003): "Adult outcomes for students with cognitive disabilities three-years after high-school: the impact of self-determination", Education and Training in Developmental Disabilities, $38(2)$ : I3 I-I 44 .

Winn, S. \& Hay, I. (2009): “Transition from school for youths with a disability: Issues and Challenges", Disability \& Society, 24(I): IO3-II 5 . 